\title{
Linx
}

Revue des linguistes de l'université Paris X Nanterre

$57 \mid 2007$

Études de syntaxe : français parlé, français hors de France, créoles

\section{Remarques à propos du système prépositionnel de l'acadien en Nouvelle-Écosse}

Julia Hennemann

\section{OpenEdition}

\section{Journals}

Édition électronique

URL : http://journals.openedition.org/linx/74

DOI : $10.4000 /$ linx.74

ISSN : 2118-9692

Éditeur

Presses universitaires de Paris Nanterre

Édition imprimée

Date de publication : 1 décembre 2007

Pagination : 79-88

ISSN : 0246-8743

Référence électronique

Julia Hennemann, «Remarques à propos du système prépositionnel de l'acadien en NouvelleÉcosse », Linx [En ligne], 57 | 2007, mis en ligne le 15 février 2011, consulté le 15 mai 2020. URL http://journals.openedition.org/linx/74 ; DOl : https://doi.org/10.4000/linx.74 


\title{
Remarques à propos du système prépositionnel de l'acadien en Nouvelle-Ecosse
}

\author{
Julia Hennemann \\ Université de Regensburg (Allemagne)
}

\section{Introduction}

Le but de cet article est de donner un aperçu de quelques phénomènes du système prépositionnel du français acadien tel qu'il est parlé à l'Isle Madame en NouvelleEcosse. En étudiant ainsi la morphosyntaxe d'un de ces français dits «marginaux », on espère dégager des informations notamment sur le français parlé aux $17^{\mathrm{e}}$ et $18^{\mathrm{e}}$ siècles, quand les premiers colons s'installèrent dans cette région de la Nouvelle-France. Un regard sur quelques créoles à base lexicale française peut également nous aider dans notre analyse, car ils se sont développés vers la même période, mais sous des conditions socioculturelles complètement autres. On fait l'hypothèse que des évolutions linguistiques que l'on peut aussi observer à leurs débuts dans l'acadien s'y sont accélérées.

A partir d'un corpus constitué d'échanges informels, recueilli à l'Isle Madame en 2005 et 2007, nous présenterons des prépositions simples et des locutions prépositives, surtout à valeurs locale et temporelle, qui montrent des écarts par rapport au français de France ${ }^{1}$.

\footnotetext{
${ }^{1}$ Par «français de France », nous entendons à la suite de Thibault, « le français tel qu'il est parlé par la plupart des Français aujourd'hui » (cf. Thibault $2006: 239$ ).
} 
Finalement, cette étude du système prépositionnel devrait apporter des informations sous un angle comparatif, qui seront utiles pour situer la variété acadienne de l'Isle Madame dans le continuum ${ }^{2}$ des français plus ou moins « avancés » d'outre-mer, dans le cadre d'une étude des spécificités morphosyntaxiques du parler acadien de l'Isle Madame.

Nous présenterons d'abord une brève vue d'ensemble de la situation géographique et sociolinguistique de l'Isle Madame, avant d'exposer notre cadre méthodologique. Puis nous détaillerons les types d'écarts que l'on peut observer dans le système prépositionnel par rapport au français standard, à partir de quelques prépositions et de leur emploi spécifique en acadien. Finalement, nous montrerons quelques tendances générales du système morphosyntaxique du parler acadien.

\section{Situation géographique et sociolinguistique}

Avant de plonger dans les structures grammaticales du parler acadien à l'Isle Madame, il faut présenter quelques faits généraux sur ce parler et dépeindre la situation sociolinguistique de ses locuteurs dans la province de la Nouvelle-Ecosse. L'Isle Madame est située à l'entrée du Cap Breton, et elle est reliée à la terre ferme par un pont. L'île est ainsi nommée en l'honneur de la deuxième épouse de Louis XIV, Françoise d'Aubigné, la future Madame de Maintenon.

A part la région de Pubnico dans le sud-ouest, l'Isle Madame est la seule région parmi les isolats acadiens de Nouvelle-Ecosse qui a pu être repeuplée par des Acadiens après l'événement décisif de leur histoire, la Déportation entre 1755 et 1763. C'est pourquoi on parle de "continuité géographique » dans la temporalité (Flikeid (1997: 258) : "geographic continuity »). Aujourd'hui, le français continue d'y être parlé, bien qu'il soit de plus en plus concurrencé par l'anglais. Selon les chiffres démolinguistiques des données du recensement de 2006, 47,3\% des habitants (1.635 sur 3.455) ont le français pour langue maternelle. Ils sont encore 1.050 à déclarer le français comme la langue la plus souvent parlée à la maison ${ }^{3}$. Ces chiffres ne laissent qu'à peine entrevoir le considérable repli du français dans la vie quotidienne. La baisse est plus visible lorsqu'on compare les taux d'assimilation ${ }^{4}$ : alors qu'en 1991, ce taux s'élevait à seulement 0,01 pour la Baie Sainte-Marie et 0,14 pour Chéticamp, il était déjà de 0,31 à l'Isle Madame (cf. Flikeid 1997 : 263). Aujourd'hui, les chiffres se sont accentués : en 2006, jusqu'à 0,36 pour l'Isle Madame. On est donc face à une assimilation linguistique

\footnotetext{
${ }^{2}$ La notion de «continuum » est contestée dans ce contexte, cf. Neumann-Holzschuh / Wiesmath (2006).

${ }^{3}$ Cf. http://www12.statcan.ca/census-recensement/2006/dp-pd/prof/92-591/details/Page.cfm?Lang $=\mathrm{E} \& \mathrm{Geo} 1=\mathrm{CSD} \&$ Code $1=1216001 \& \mathrm{Geo} 2=\mathrm{PR} \&$ Code $2=12 \& \mathrm{Data}=$ Count $\&$ Search Text $=$ Richmond $\% 20$ Subd. $\% 20 \mathrm{C} \&$ SearchType $=$ Begins $\&$ SearchPR $=01 \& B 1=$ All \&Custom $=$, consulté le 16 décembre 2008.

${ }^{4}$ Le taux d'assimilation se calcule de la manière suivante: (nombre de personnes ayant appris le français comme langue maternelle moins nombre de personnes parlant le français à la maison) divisé par nombre de personnes ayant appris le français comme langue maternelle.
} 
croissante de la population francophone, qui ne peut apparemment plus être endiguée ou retardée par l'instauration d'un système scolaire complètement francophone de la maternelle à la douzième classe.

Comment ces données sociolinguistiques se manifestent-elles dans les caractéristiques du parler ? On rencontre des faits archaïques et/ou dialectaux sauvegardés à travers des siècles d'isolement quasi-total, contrairement à ce qui s'est passé dans d'autres régions francophones (Québec, France métropolitaine etc.): ils ont par conséquent échappé à toute entreprise normative. De plus, on observe également bon nombre de phénomènes dus au contact linguistique entre le français et l'anglais, un contact particulièrement intense dans le nord-est de la Nouvelle-Ecosse.

\section{Cadre méthodologique}

Notre étude morphosyntaxique est fondée sur un corpus dont la source principale est une série d'entrevues effectuées par nos soins pendant un premier séjour de juillet à novembre 2005 (durée totale d'environ 50 heures) en Nouvelle-Ecosse. Il s'agit principalement de conversations aussi informelles que possible, qui manifestent quand même des différences entre locuteurs parlant un acadien traditionnel (désormais l.a.) et locuteurs parlant un français très proche du standard (l.s.). A l'Isle Madame, nous étions souvent accompagnée de la mère de notre famille d'accueil, âgée de 74 ans à l'époque. Ainsi, nous n'étions parfois que simple observatrice. Les thèmes de la conversation étaient assez spontanés dans le contexte, surtout souvenirs d'enfance, traditions acadiennes et parcours de vie. Aux transcriptions de ces entrevues, on a ajouté des énoncés recueillis dans les familles d'accueil lors d'un deuxième séjour en 2007.

Notre analyse sera différentielle: ce sont les écarts par rapport au français de France qui nous intéressent. Elle sera en outre comparative : là où cela s'avérera intéressant, nous effectuerons des comparaisons avec des corpus recueillis pour d'autres variétés de français (autres régions en Nouvelle-Ecosse, ensemble des provinces maritimes, québécois), et les créoles.

\section{4. Écarts par rapport au français de France}

Commençons par noter quelques particularités du système prépositionnel à travers deux écarts phonétiques, chus et sus, écarts oraux que nous présentons ici de façon graphique et non en phonétique ${ }^{5}$ :

${ }^{5}$ Quelques explications sur la transcription des entrevues: nous adaptons les modalités de transcription de Ehlich (1993) à nos besoins :

- des mots empruntés à l'anglais et gardant leur prononciation anglaise sont en majuscules, p. ex. IMPLANT.

- des verbes empruntés à l'anglais mais intégrés morphologiquement sont en italiques : p. ex. landait.

- les formes tout, toute, tous et toutes sont transcrits soit tout soit touT, selon leur prononciation.

- une barre oblique directement derrière une lettre, sans espace, indique que le mot a été interrompu, p. ex. e/ ou $c h /$. 
Julia Hennemann

[1] J'ai té sus le docteur, l'autre jour, sus le médecin pis i m'a dit I WAS IN PERFECT HEALTH. (ILM, E1) ${ }^{6}$

[2] il était chus eux la semaine qu'al e/ qu'a / al accouchait pour ch/ pour son garçon. (ILM, E10)

Concernant la première particularité phonétique, on observe que sur n'existe que chez les locuteurs les plus standardisants ; sus est une forme qui était en usage en français jusqu'au 17e siècle (cf. Brunot/Bruneau 1949: 439), demeurée courante dans certains dialectes français, mais aussi au Canada, en Louisiane et à Saint-Pierre et Miquelon (cf. Brasseur 2001 : 435). La deuxième préposition, chus, est dans notre corpus la variante combinatoire de sus devant des pronoms personnels disjoints. Alors que dans d'autres régions, chus s'emploie également devant des syntagmes nominaux et des noms propres (cf. par exemple Arrighi 2005: 131 et 160 pour le NouveauBrunswick; et p. 252 pour l'Ile du Prince Edouard), tel n'est pas le cas dans notre corpus, où règne au contraire une stricte différenciation entre les deux variantes phonétiques.

D'un point de vue morphologique, la préposition dans présente un aspect intéressant à l'Isle Madame : à part la forme dans elle-même, on trouve de nombreuses attestations où le syntagme prépositionnel « d'un + substantif » signifie sans le moindre doute « dans un... » :

[3] $\mathrm{Al}$ a eu une IMPLANT d'un œil, hein, pis et pis, si c'est du SALT, pis qu'i y a du blanc qui reste sus le butin, on voit pas. (ILM, E4)

[4] Pis il avait tou'T ça d'un sac. (ILM, E4)

Pourquoi faire l'hypothèse que d'est ici un allomorphe de la préposition dans ? Pourquoi n'aurions-nous pas affaire à la préposition de, réduite à d' devant voyelle? D'abord, de n'est attesté - à notre connaissance - nulle part dans les grammaires historiques avec le sens de $d_{a n s}{ }^{7}$. Il ne pourrait donc pas s'agir d'une acception archaïque. De plus, il serait peu probable qu'une préposition comme de, presque totalement vide de sémantisme propre, adopte tout à coup un sens local précis, sans contrainte apparente du système linguistique. Enfin, nous trouvons comme «preuve

- $\quad$ une barre oblique et un espace derrière un mot signifie qu'il y a une rupture dans l'énoncé, p. ex. qu'a / al accouchait...

- des mots lexicalisés, apparaissant comme entrée dans des dictionnaires acadiens, sont adoptés tels quels, p. ex. pis, asteure.

La phonétique ne joue en général aucun rôle dans notre analyse, et n'est par la suite pas prise en considération dans la transcription.

Les mots en gras dans les exemples représentent les phénomènes morphosyntaxiques traités.

${ }^{6}$ Conventions de notation des entrevues : successivement lieu de l'entrevue (par ex. PUB $=\underline{\text { Pubnico, }}$

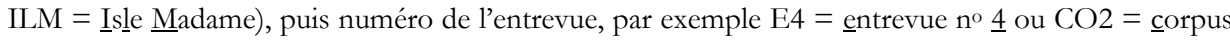
oral $n^{\circ} \underline{2}$.

${ }^{7}$ On trouve cependant $d e$ introduisant une durée (un sens donc proche du dans temporel) en ancien français (voir Godefroy, Greimas) : Il n'en out de treis iurz ne de treis nuiz de pain mangied, ne beud (Livre des Rois). 


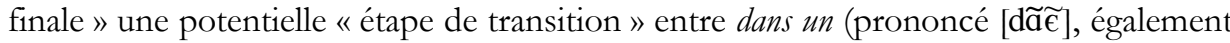

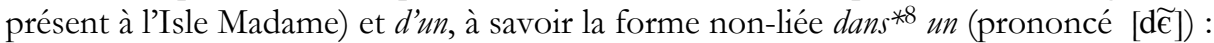

[5] S'avait été dans* un ville, ça arait té différent BUT un petit village, tout le monde se connaissait pis... (ILM, E4)

[6] Pis al a dix personne dans* un nouveau groupe. (ILM, E5)

Néanmoins, il semble qu'à un niveau qualifié de "très ordinaire », la non-liaison entre dans et un soit également possible en français parlé de France (c. p. de Françoise Gadet). Mais ces deux dernières étapes (dans* un, d'un) ne se rencontrent pas seulement avec l'article indéfini masculin $u n$, mais aussi avec la forme féminine de l'article indéfini une:

[7] Pis t'sais, quand t'es six filles dans* une famille pis les garçons te galopent touT, ... (ILM, E10)

[8] Moi, c'est / c'est j'aime mieux les mettre à l'aise. Pis même Cayouche $\mathrm{i}$ chante deux ou trois chansons anglaises qu'i les mettra à l'aise aussi. [...] Ben surtout quand-ce t'es d'une ${ }^{9}$ région comme la nôtre. (ILM, E8)

Même si nous n'avons pas encore d'explication concluante pour l'étape de nonliaison entre dans et l'article indéfini, on peut se demander si la raison de l'émergence de d'un pourrait être le « souci » d'éviter l'hiatus à la frontière de deux mots successifs, ou tout simplement une crase. Nous avons par ailleurs trois exemples dans le corpus qui pourraient conduire à postuler une quatrième étape, à savoir la réanalyse de $d^{\prime}(<$ dans) en $d e$ :

[9] ... i y a des choses qui s'est passées j'tais t-êt pas totalement d'accord de notre f/ milieu parce qu'on avait grandi jusqu'à certains points. (ILM, E8) ${ }^{10}$

[10] Et là, ils travaillent du mois de mai. Mais elle, je crois qu'elle a commencé dans* avril. (ILM, E5) $)^{11}$

[11] Et pis mon grand-père Samson est mort des soixante, dix-neuf cent soixante-... (ILM, E4)

L'exemple 9 montre que $d e$, dans sa forme non-amuïe, s'utilise également dans le sens de dans. Il peut même faire l'objet d'un nouvel amalgame complet, selon l'exemple de la préposition homophone $d e$ : ainsi $d e+l e$ devient $d u$ ("dans le »)

\footnotetext{
${ }^{8}$ Ici, l'astérisque $\left(^{*}\right)$ indique la non-liaison entre le $s$ final et la voyelle initiale du lexème suivant.

${ }^{9}$ Le contexte de cet énoncé permet de considérer sans aucun doute que la signification est « dans », et non une indication d'origine.

10 Après avoir réécouté l'enregistrement à plusieurs reprises, nous excluons la possibilité que de notre milieu dépende de être d'accord. L'incise j'étais pas totalement d'accord nous semble plutôt à interpréter comme une subordonnée relative avec omission du pronom relatif qui, en français standard, serait dont ou avec lesquelles : «il y a des choses qui s'est passées dont j'étais pas totalement d'accord / dans notre milieu... ».

${ }^{11}$ Le syntagme prépositionnel habituel en acadien est plutôt : « dans le mois de ... ».
} 
[cf. exemple 10] et de + les devient des («dans les ») [cf. exemple 11]. Nous pouvons résumer ces quatre étapes de la manière suivante :

\begin{tabular}{|c|c|c|c|c|}
\hline étape & $1^{\text {ère }}$ & $2^{\mathrm{e}}$ & $3^{\mathrm{e}}$ & $4^{\mathrm{e}}$ \\
\hline & dans un $(e)$ & dans*un(e) & d'un $(e)$ & de = « dans » \\
\hline $\begin{array}{c}\text { attestations dans le } \\
\text { corpus }\end{array}$ & $\begin{array}{c}6+23 \\
(=\mathrm{la}+\mathrm{les})\end{array}$ & 10 & 12 & 3 \\
\hline
\end{tabular}

Tableau : développement de dans + article indéfini

Même si notre corpus n'est pas assez vaste pour tirer des conclusions définitives, nous pouvons tout de même observer que les Acadiens de l'Isle Madame parlant un acadien plutôt traditionnel ont une nette préférence pour les formes non-liées (étape 2) et la forme amalgamée (étape 3). Même si la forme standard dans un(e) est encore présente dans quelques énoncés, la réanalyse de $d^{\prime}(<$ dans) en de montre que l'évolution va dans le sens des étapes 3 et 4 .

Nous n'avons trouvé aucune référence - standard ou non - de ce phénomène, ce qui pourrait bien être un indice, mais pas forcément une preuve. Pour les autres régions francophones en Amérique du Nord, les différentes étapes de ce phénomène ne sont attestées que de manière sporadique. Starets a pu observer une «amalgamation» dans son étude du langage des enfants dans plusieurs écoles néo-écossaises (cf. Starets 1986 : 503). Papen/Rottet (1997: 103) constatent pour la Louisiane que dans est souvent prononcé [d] quand il est suivi d'un article indéfini. Mises à part ces attestations, il apparait difficile de retrouver une éventuelle étape intermédiaire, car quelques-uns des corpus consultés ne sont pas transcrits phonétiquement, ou bien les sources sonores ne sont que difficilement disponibles.

Finalement, nous trouvons aussi des écarts lexicaux (comme dans le mitan de $e^{12}$ « au milieu de », cf. exemple 12) ou des différences sémantiques (extension sémantique de à travers de qui peut aussi signifier « en face de » à l'Isle Madame, cf. exemple 13) :

[12] [à propos de sa date de naissance] Oui, dans le mitan de la guerre, oui, oui. (PUB, E4)

[13] Au SENIOR CLUB à travers du LIQUOR [STORE]. (ILM, E1)

\footnotetext{
12 Cormier (1999 : article mitan) : «Héritage de France ; attesté en français sous la forme actuelle dès le XIII e s[iècle]; puis de nos jours avec les marques 'vieilli' ou 'vieux', 'dialectal' ou 'régional' et 'populaire'».
} 


\section{Deux phénomènes innovants?}

\section{1. clair (de)}

Voyons à présent deux spécificités du système prépositionnel: nous avons repéré dans le corpus une préposition qui n'est attestée que dans très peu de variétés du français, à savoir clair (de):

[14] Eux-autres, ils sont venus là. Comme mon patron il est venu après dix-sept ans clair de la région. (ILM, E3)

[15] On peut pas aller clair du Canada WITHOUT A PASSPORT. (ILM, CO2)

[16] on était pas loin, un mille clair la terre (ILM, CO2)

On trouve aussi la tournure se tenir clair de dans notre corpus :

[17] I dit : tenez-vous clair de c'tés docteurs là. Tenez-vous clair de c'tés gars-là. (ILM, E1)

Il ne s'en trouve aucune attestation dans les travaux de L. Arrighi (2005) (NB, IPE, NÉ), de R. Wiesmath (2006) (NB), ni dans le corpus de C. Stäbler (1995) (LOU). De plus, cette grammaticalisation probable de l'adjectif clair ne se trouve pas non plus dans le FEW de Wartburg. Le seul dictionnaire dans lequel nous avons trouvé une telle entrée est celui de Brasseur pour Terre-Neuve : il cite quatre acceptions dont deux correspondent au sens de la préposition dans notre corpus : «en dehors de » et «au large de, à l'écart de » (cf. Brasseur 2001 : 114). De manière générale, on peut constater certains points communs entre le parler acadien à l'Isle Madame et le parler acadien à Terre-Neuve (cf. Brasseur 1996 : 303). Pourtant, la question reste ouverte quant à un rapport entre les attestations de clair (de) à l'Isle Madame et à Terre-Neuve. Une autre interprétation pourrait être le contact intense des deux parlers avec l'anglais. Brasseur cite la tournure clear of doing something (« exept for, apart from») qui aurait pu être transformée et connaitre ensuite une extension de sens. Mais il semble qu'on puisse, plus simplement, faire le lien avec des emplois 'spatiaux' de clear (of) : voir dans le Merriam-Webster le sens of de clear ("free from entanglement or contact") et les exemples <staying clear of controversy $>$ et surtout $<$ keep clear of the boundary $>13$. (c. p.) Néanmoins, clair de a aussi été relevé avec les significations «sans» au Québec, «loin de» ailleurs en Acadie, et «au large» aux Iles-de-la-Madeleine (cf. Brasseur 2001 : 114).

\section{2. près de, à côté de}

Une étude onomasiologique des prépositions et locutions prépositives exprimant le sens de "près de, à côté de » constitue un autre sujet intéressant, qui reflète bien les effets diversifiés se manifestant dans l'acadien de l'Isle Madame. Nous présentons dans un premier temps une liste des prépositions qui peuvent indiquer un sens similaire à « à côté de, près de » dans notre corpus :

13 Cf. http://www.merriam-webster.com/dictionary/clear, consultation le 2 octobre 2009. 
Julia Hennemann

[18] Si on allait manger, il sauvait une place à côté de lui. (ILM, E5)

[19] Pis i y avait une tou' $\mathrm{T}$ p'tiT chambre en bas pour le père pis la mère auprès de la cuisine... (ILM, E9)

[20] tu t'embrassais pas beaucoup auprès tes parents, parce que [...] Oui. Ben il étiont un peu stricts. (ILM, E1)

[21] Pis i y en avait une autre en haut de sus Suzanne là, juste au côté d'école ${ }^{14}$ qu'on était fréquenter nous-autres. (ILM, E9)

[22] Bentôt D. B. ça rentre, son homme, son mari est dans la cuisine, PASSED OUT, évanoui. Evanoui au ras R. pis nous-autre j’avions sa femme je croyions qu'al allait mourir. (ILM, E1)

[23] Nous-autres, si j'avions un BOYFRIEND ma mère s'assisait au s/ au ras de nous-autres avec une lampe à KEROSENE. (ILM, E1)

[24] Et pis, $\mathrm{m} / \mathrm{ma}$ femme m'a huché parce qu'a voit pas trop à bord du chemin, hein ? (ILM, E4) [Contexte : la femme, qui voit mal, est assise à côté de la fenêtre, voit quelqu'un dans la cour, et demande s'il s'agit des éboueurs]

[25] i y avait la chambre / la lit était NEXT la cuisine. (ILM, E10)

[26] A me landit sus NEXT DOOR la salle, tu sais. (ILM, E4)

[27] Ah tu / ah, t'as vnu ici, droète NEXT DOOR du cimetière là ? NEXT DOOR du cimetière. (ILM, E2)

[28] la femme NEXT de moi, la femme NEXT DOOR (ILM, CO2)

Il y a donc onze possibilités pour exprimer le sens de "près de ", même si toutes ces variantes ne sont pas simplement interchangeables. Nous donnerons maintenant quelques brèves explications concernant les variantes présentées ci-dessus.

Les formes du français standard, à côté de [cf. exemple 18] et près de, sont rares, voire inexistantes à l'Isle Madame. Les deux occurrences de à côté de émanent de la même locutrice, qui a été pendant longtemps en contact avec le français standard. En ce qui concerne près de, Wiesmath confirme aussi pour son corpus du sud-est du Nouveau-Brunswick que cette préposition présente une fréquence relativement basse (cf. Wiesmath 2006 : 248). Arrighi va dans le même sens, en se référant à Chaudenson qui «fait remarquer que 'les créoles n'ont pas de trace, en général, de la préposition 'près de', il indique que près de n'apparaît sans doute que peu dans l'usage courant, même actuel du français ordinaire » (Arrighi 2005 : 362).

On trouve des locutions prépositives très proches, comme auprès (de) [cf. exemples 19 et 20] et au côté de [cf. exemple 21], qui sont plus fréquentes. C'est aussi la forme opré qui s'est imposée par exemple en créole guadeloupéen ${ }^{15}$. Passons à une

\footnotetext{
${ }^{14}$ L'élision de l'article défini est plutôt atypique du parler acadien à l'Isle Madame. On en trouve des cas surtout en Louisiane (cf. p. ex. Dajko 2009 : 192).

15 [a] Vin opré mwen pou mwen di ou sa! (Viens près de moi, que je te dise quelque chose!) (Tourneux/ Barbotin 1990 : article oprê).
} 
autre variante, plutôt typique de l'acadien traditionnel, qui se rencontre dans presque tous les parlers français en Amérique du Nord, à savoir au ras (de) [cf. exemples 23 et 24]. Dans la plupart des cas, les locuteurs de l'Isle Madame utilisent la locution prépositive sans de, c'est donc la même observation que celle de Wiesmath pour le Nouveau-Brunswick (cf. Wiesmath 2006 : 248). Cette forme continue à exister par exemple en créole guadeloupéen où l'on trouve ora ${ }^{16}$ et owa. A bord de [cf. exemple 24], qui n'a d'ailleurs qu'une seule attestation dans notre corpus, s'y trouve également sous la forme $\grave{o} b \hat{o}^{17}$. Finalement, nous constatons des variantes créées à partir des mots anglais NEXT ou NEXT DOOR [cf. exemples 25-28]. NEXT a peut-être pris le détour de l'intégration comme adjectif avant de devenir un moyen prépositif. Dans le corpus de Wiesmath par exemple, on ne trouve en effet jusqu'à présent que la fonction adjectivale de NEXT. ${ }^{18}$

Pour tirer des conclusions de cette analyse onomasiologique, commençons par la fréquence : près (3 attestations), côté (6), bord (1), au ras (7), NEXT (DOOR) (6). On voit donc que les formes contenant côté, au ras et NEXT (DOOR) sont de loin les plus fréquentes. Ici, on pourrait se référer à des processus autorégulateurs qui vont du sémantiquement vide au sémantiquement plein, ou de l'opaque au transparent (cf. Chaudenson/ Mougeon/Beniak 1993 : 28) : au lieu de près de, les locuteurs préfèrent des formes avec bord ou bien se servent de mots de la langue omniprésente, l'anglais, comme NEXT (DOOR) dont le sens est plus transparent. La forme traditionnelle au ras en revanche est tellement ancrée dans l'usage qu'elle parvient à résister à cette tendance.

16 [b] Kabwa-la néyé ora lésyé. (La charrette est embourbée jusqu’à l'essieu.) (Tourneux/ Barbotin 1990 : article ora).

17 [c] I rivé òbò kaz-la. (Il arriva près de la maison.) (Tourneux / Barbotin 1990 : article òbò).

18 Voilà une comparaison des catégories des occurrences anglaises dans le parler de l'Isle Madame avec les résultats de Flikeid (1989 : 196) pour Pubnico et Chéticamp et de Poplack et al. (1988: 63) pour Ottawa-Hull (tableau de Flikeid 1989: 196):

\begin{tabular}{|l|r|r|r|r|}
\hline & Pubnico & Chéticamp & Ottawa-Hull & Isle Madame \\
\hline Noms & $61 \%$ & $67 \%$ & $64 \%$ & $68 \%$ \\
\hline Verbes & $19 \%$ & $18 \%$ & $14 \%$ & $15 \%$ \\
\hline Adjectifs & $9 \%$ & $9 \%$ & $8 \%$ & $6 \%$ \\
\hline Adverbes & $6 \%$ & $2 \%$ & $<1 \%$ & $5 \%$ \\
\hline Prépositions & $1,5 \%$ & $<1 \%$ & $<1 \%$ & $1,4 \%$ \\
\hline Pronoms & $1 \%$ & $<1 \%$ & $<1 \%$ & $<1 \%$ \\
\hline Conjonctions & $1 \%$ & $<1 \%$ & $1,5 \%$ & $<1 \%$ \\
\hline Interjections & $2,5 \%$ & $3,5 \%$ & $12 \%$ & $2,39 \%$ \\
\hline
\end{tabular}


Julia Hennemann

\section{Tendances et premiers résultats}

Dans cette analyse assez succincte, nous avons pu mettre en lumière quelques détails du système prépositionnel. Il ressort, de façon plus nette que pour d'autres domaines de la morphosyntaxe, la coexistence d'un grand nombre de variantes qui expriment plus ou moins le même sens en fonction du contexte. On trouve des formes traditionnelles, des formes standard apparues durant les dernières décennies, après des contacts plus intenses avec le français international. Mais, surtout à l'Isle Madame, on rencontre - même dans le système prépositionnel qui est dit plus résistant aux anglicismes que d'autres parties de la langue - des variantes formées à partir d'expressions anglaises.

Les cas de grammaticalisation de clair de ainsi que le développement de dans un montrent aussi qu'il ne s'agit pas d'un système stabilisé. 


\section{RÉFÉRENCES BIBLIOGRAPHIQUES}

ARRIGHI, L. (2005), Etude morphosyntaxique du français parlé en Acadie. Une approche de la variation et du changement linguistique en français. Thèse de doctorat non publiée, U. d'Avignon.

Brasseur, P. (1996), "Changements vocaliques initiaux dans le français de Terre-Neuve", in Lavoie, Th. (éd.), Français du Canada - français de France. Actes du quatrième colloque international de Chicoutimi. Tübingen : Niemeyer, 295-305.

BRASSEuR, P. (2001), Dictionnaire des régionalismes du français de Terre-Newve. Tübingen : Max Niemeyer Verlag.

Brunot, F./Bruneau, CH. (1949), Précis de grammaire historique de la langue française. Paris: Masson et Cie.

Chaudenson, R./Mougeon, R./Beniak, É. (1993), Vers une approche panlectale de la variation du français. Paris : Didier.

CORMIER, Y. (1999), Dictionnaire du français acadien. Montréal : Fides.

DajKo, N. (2009), Ethnic and geographic variation in the French of the Lafourche Basin. Thèse de doctorat. New Orleans : Tulane University.

EHLICH, K. (1993), «HIAT : A Transcription System for Discourse Data », in Edwards, J. A./ Lampert, M. D.: Talking data: transcription and coding in discourse research. Hillsdale: Lawrence Erlbaum Associates, 123-148.

FLIKEID, K. (1989) : «'Moitié anglais, moitié français'? Emprunts et alternance de langues dans les communautés acadiennes de la Nouvelle-Ecosse", Revue québécoise de linguistique théorique et appliquée 8, 177-228.

FLIKEID, K. (1997), «Structural Aspects and Current Sociolinguistic Situation of Acadian French », in Valdman, A. (éd.), French and Creole in Louisiana. New York, London: Plenum Press, 255-286.

Neumann-HolzschuH, I./Wiesmath, R. (2006), «Les parlers acadiens: un continuum discontinu », Revue canadienne de linguistique appliquée/Canadian Journal of Applied Linguistics, 9, 233-249.

PAPEN, R./RotTET, K. (1997), «A structural sketch of the Cajun French spoken in Lafourche and Terrebonne Parishes ", in Valdman, A. (éd.), French and Creole in Louisiana. New York, London : Plenum Press, 71-108.

Poplack, S. (1988), «The Social Correlates and Linguistic Processes of Lexical Borrowing and Assimilation », Linguistics 26, 47-104.

STÄBLER, C. (1995), La vie dans le temps et asteur. Ein Korpus von Gesprächen mit Cadiens in Louisiana. Tübingen: Narr.

STARETS, M. (1986), Description des écarts lexicaux, morphologiques et syntaxiques entre le français acadien des enfants acadiens néo-écossais et le français standard. Québec : CIRB. 
Julia Hennemann

Thibault, A. (2006), Compte rendu de Valdman, A./Auger, J./Piston-Hatlen, D. (éds.), Le français en Amérique du Nord. État présent, Québec : Les Presses de l'Université Laval, 2005, Revue de linguistique romane 70 (2006), 238-250.

Tourneux, H. / Barbotin, M. (1990) : Dictionnaire pratique du créole de Guadeloupe (Marie-Galante) suivi d'un index français-créole. Paris : Karthala et al.

Wiesmath, R. (2006), Le français acadien. Analyse syntaxique d'un corpus oral recueilli au NonveauBrunswick / Canada. Paris : L'Harmattan. 